\title{
Particle-size segregation in dense granular avalanches
}

\author{
J.M.N.T. Gray ${ }^{a, *}$, P. Gajjar ${ }^{a}$, B.P. Kokelaar ${ }^{b}$ \\ ${ }^{a}$ School of Mathematics and Manchester Centre for Nonlinear Dynamics, \\ University of Manchester, Manchester, M13 9PL, UK \\ ${ }^{b}$ Department of Earth and Ocean Sciences, University of Liverpool, Liverpool, L69 3GP, UK
}

\begin{abstract}
Particles of differing sizes are notoriously prone to segregate, which is a chronic problem in the manufacture of a wide variety of products that are used by billions of people worldwide every day. Segregation is the single most important factor in product non-uniformity, which can lead to significant handling problems as well as complete batches being discarded at huge financial loss. It is generally regarded that the most important mechanism for segregation is the combination of kinetic sieving and squeeze expulsion in shallow granular avalanches. These free-surface flows are more common than one might expect, often forming part of more complicated flows in drums, heaps and silos, where there is mass exchange with underlying regions of static or slowly moving grains. The combination of segregation and solid-fluid granular phase transitions creates incredibly complicated and beautiful patterns in the resulting deposits, but a full understanding of such effects lies beyond our capabilities at present. This paper reviews recent advances in our ability to model the basic segregation processes in a single avalanche (without mass exchange) and the subtle feedback effects that they can have on the bulk flow. This is particularly important for geophysical applications, where segregation can spontaneously self-channelize and lubricate the flow, significantly enhancing the run-out of debris-flows, pyroclastic flows, rock-falls and snow-slab avalanches.
\end{abstract}

Keywords: avalanches, granular materials, particle size-segregation, particle mixing, run-out

\section{Introduction}

Shallow granular free-surface flows (granular avalanches) are one of the most common styles of particle transport in industrial bulk solids handling. They form in chute flows $[1,2]$ and occur as thin fluid-like layers at the free-surface of heaps $[3,4]$, silos $[5,6]$ and rotating tumblers [7-9]. It is precisely these avalanches that 5 are extremely efficient at segregating particles by size [10, 11]. The British Materials Handling Board "User guide to segregation" [5] estimate that the UK processes 165 million tonnes of bulk chemicals, detergents, pharmaceuticals, plastics, glass, minerals and food stuffs each year. In the US it is over 1 trillion tonnes [12], so total world production is huge especially when the major mining nations are included. The British Materials Handling Board [5] also state that "segregation is the most influential common factor which adversely affects the uniformity of bulk materials, raising problems of product suitability and also giving rise to many types of handling and processing difficulties within the manufacturing plant". An improved understanding of segregation therefore has the potential to affect the manufacture of many ordinary consumer products that are used by billions of people throughout the world every day.

It is generally regarded that the dominant mechanism for segregation is the combination of kinetic sieving $[13,14]$ and squeeze expulsion [10]. Savage and Lun [10] argued that the avalanche acts as a "random fluctuating sieve" in which small particles are statistically more likely to fall down into gaps as they are sheared down slope. This mechanism is complemented by "squeeze expulsion", which levers all particles

\footnotetext{
${ }^{*}$ Correspondence and reprints.

Email addresses: nico.gray@manchester.ac.uk (J.M.N.T. Gray), parmesh.gajjar@alumni.manchester.ac.uk (P. Gajjar), P.Kokelaar@liverpool.ac.uk (B.P. Kokelaar)
} 

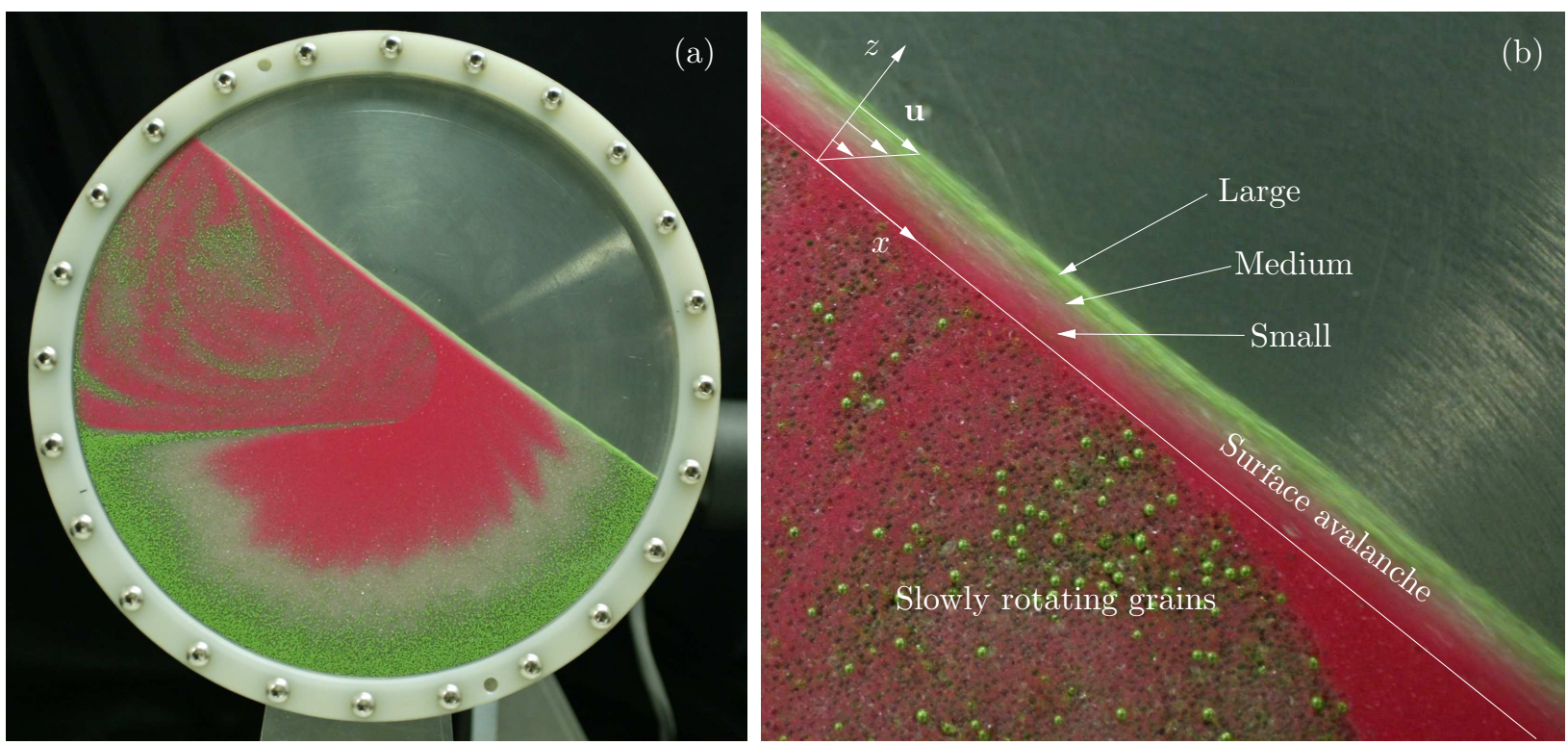

Figure 1: Segregation of a ternary mixture composed of 500-750 $\mu \mathrm{m}$ (green), 400-500 $\mu \mathrm{m}$ (white) and 75-150 $\mu \mathrm{m}$ (pink) particles in a rotating drum (a). All the segregation occurs in a thin surface avalanche (b) which transforms the initially homogeneous mixture into a radial segregation pattern (a) with small particles deposited first and the large ones last [Gray, J.M.N.T. \& Ancey, C. (2011) Multi-component particle-size segregation in shallow granular avalanches, J. Fluid Mech. 678, 535-588. Reproduced with permission, 44].

upwards, resulting in a net flux of small particles to the base and large grains to the surface of the flow. Fan and Hill [15] have put forward an alternative hypothesis that, in dense systems, large particles are 20 driven to regions of higher granular temperature by kinetic stress gradients, which results in them rising to the surface of the avalanche in a rotating drum [16] or being driven to the sidewalls in a vertical chute flow [15]. The problem can also be viewed as one of lift and drag forces acting on a large particle in an effective fluid medium of fine particles [17-19].

Whatever the precise micro-mechanical mechanism, the fact remains that granular avalanches are very efficient at sorting particles to produce an "inversely graded" size distribution that coarsen upwards [13, 14]. Such segregation also occurs in geophysical mass flows such as debris-flows [20-23], dense pyroclastic avalanches [24-26], lahars [27, 28], rockfalls [29] and snow avalanches [30-32]. When combined with erosion and deposition, segregation within these flows can produce incredibly complex deposits that geologists concerned with natural hazards are keen to understand in order to infer properties of the parent flows. The 30 particle-size segregation can have a subtle feedback on the bulk flow itself, with the spontaneous formation of levees and channels that enhance run-out [22, 33-39]. Improving our knowledge of segregation is therefore not only important for the manufacture of many bulk solids [40-42], but also for proper assessment of hazards and hence risks associated with geophysical flows [24, 43].

A typical example of the effect of segregation in a surface avalanche is shown in figure 1(a). The vast majority of grains are in solid body rotation, but there is a very thin fluid-like avalanche at the freesurface (Fig. 1b) where all the segregation takes place. In this case the granular material is composed of large $500-750 \mu \mathrm{m}$ (green), medium 400-500 $\mu \mathrm{m}$ (white) and small 75-150 $\mu \mathrm{m}$ (pink) particles, that coarsen upwards (inversely grade) in the surface avalanche. This has a major influence on the resulting particle-size distribution in the rotating deposit. Grains are entrained into the avalanche on the upper part of the slope

40 and are continuously deposited on the lower half. Since the small particles segregate to the base of the avalanche they are the first to be deposited, with the medium sized ones next and finally the largest grains depositing near the drum wall. As a result, even just a partial rotation of the drum causes an initially homogeneous mixture (on the left of Fig. 1a) to develop into a pronounced radial size distribution (on the right of Fig. 1a). The combination of particle-size segregation in surface avalanches and granular fluid-solid 
45 phase transitions creates amazingly complicated and beautiful patterns and deposits [7, 8, 24, 45].

Despite many years of research, particle size segregation continues to present considerable practical problems in many industrial processes [46, 47]. Sometimes the segregation is useful, such as in the mining industry [48], but mostly it is undesired and degrades the quality of products leading to increased costs and wastage. The behaviour and consequences of avalanche segregation has not yet been fully appreciated by so industry. The "User guide to segregation" [5] and a recent textbook by Schulze [6] both ascribe "trajectory segregation" at the exit of a chute to differential air drag on the particles. While very fine particles can be subject to differential drag, in most cases the real reason that the large particles travel farther than the fines at the exit of the chute is that the grains are segregated by an avalanche within the chute itself. Hence, when they emerge, the largest grains are in the fastest moving surface layers and are projected farther than the fine particles in the slower moving basal regions of the flow.

In order to mitigate the worst effects of segregation, it is important to understand the physical processes and develop predictive mathematical theories. Bridgwater et al. [49] were the first to develop a continuum model for segregation based on the idea of competition between percolation and diffusion. Their model was formulated in terms of the concentration $\phi$ of a component expressed as a fraction of the solid volume and took the form

$$
\frac{\partial \phi}{\partial t}+\frac{\partial}{\partial z}\left(q \phi(1-\phi)^{2}\right)=\frac{\partial}{\partial z}\left(D \frac{\partial \phi}{\partial z}\right)
$$

where $z$ was the coordinate parallel to gravity, $q$ was a percolation rate and $D$ was a diffusion coefficient. The segregation is achieved by the second term on the left-hand side of (1) with a segregation flux that is proportional to $\phi(1-\phi)^{2}$. This has the property that it shuts off when the concentration reaches zero and unity, which automatically keeps the concentration in the range $[0,1]$. Bridgwater et al. [49] realized that the percolation rate $q$ was dependent on the shear rate $\dot{\gamma}$, the particle size ratio $d_{s} / d_{l}$ and the normal pressure $p$, and they used shear box experiments [50-53] to investigate the functional dependence. Savage and Lun [10] used statistical mechanics and information entropy theory to derive a segregation model from first principles. Their model was formulated in terms of number densities and fluxes, so that it is difficult to see the direct relation with the model of Bridgwater et al. [49]. It consists of two mass balance equations for the large and small particles

$$
u(z) \frac{\partial \rho^{l}}{\partial x}+\frac{\partial}{\partial z}\left(\rho^{l} q_{\mathrm{NET}}^{l}\right)=0, \quad u(z) \frac{\partial \rho^{s}}{\partial x}+\frac{\partial}{\partial z}\left(\rho^{s} q_{\mathrm{NET}}^{s}\right)=0,
$$

where $z$ is the normal coordinate through the avalanche and $u(z)$ is the down stream velocity profile. The expressions for the net percolation velocities are, in general, very complicated functions of the number density and grain size ratio, however, Gray and Thornton [54] showed that in the dilute limit they are equivalent to

$$
q_{\mathrm{NET}}^{l}=q \phi^{s}, \quad q_{\mathrm{NET}}^{s}=-q \phi^{l},
$$

where $\phi^{l}$ and $\phi^{s}$ are the concentrations of large and small particles, respectively. Since the densities $\rho^{l}=\phi^{l} \rho^{*}$ and $\rho^{s}=\phi^{s} \rho^{*}$ are related to the density of the grains $\rho^{*}$ by a linear volume fraction scaling, it follows that the equivalent segregation flux is proportional to $\phi^{s} \phi^{l}$. This is similar to that of Bridgwater et al. [49] in equation (1), which in the notation of Savage and Lun [10] is proportional to $\phi^{s}\left(\phi^{l}\right)^{2}$ since $\phi^{l}=1-\phi^{s}$. Although Savage and Lun's [10] theory provided detailed functional forms for the percolation rate $q$ dependent on the particle-size ratio and the shear rate, there was no explicit dependence on gravity, which is odd since kinetic sieving is fundamentally a gravity driven process. Both Savage and Lun [10] and Bridgwater et al. [49] tried to construct exact solutions in the absence of diffusion using the method of characteristics. These were only partially successful, although later attempts have generated complete time and spatially dependent exact solutions in both uniform and non-uniform flows [54-58]. A review of the results in the absence of diffusion is provided in [47]. Savage and Lun [10] did not formulate proper jump conditions [44, 54, 59] at the concentration shocks that develop, so they underestimated the distance for an initially homogeneous mixture to segregate in a chute flow [54]. This effect is small at low-small particle concentrations so that good agreement was found between the theory and chute flow experiments in this limit [10,11]. Independently, 
Dolgunin and Ukolov [60] simply wrote down a segregation equation of the form

$$
\frac{\partial \phi}{\partial t}+\frac{\partial}{\partial x}(\phi u)+\frac{\partial}{\partial z}(q \phi(1-\phi))=\frac{\partial}{\partial z}\left(D \frac{\partial \phi}{\partial z}\right),
$$

based on the insight that the segregation flux must shut off when the large or small particles are in a pure phase, i.e. the segregation flux must be proportional to $\phi(1-\phi)$, where $\phi$ is the volume fraction of one of the components. Equation (4) combines the particle transport by the bulk velocity and the form of the segregation flux in the dilute limit used in Savage and Lun's [10] formulation, with Bridgwater et al.'s [49] diffusion and time-dependent term. Dolgunin and Ukolov's [60] model therefore contains all the essential ingredients of the bidisperse segregation models that are used today. Equation (4) has been used to model segregation due to density differences between the particles $[2,17]$ and it is closely related to models of sedimentation in suspensions $[61,62]$. The next section will examine how such a model can be derived.

\section{Mixture theory framework}

Following Gray and Thornton [54], Gray and Chugunov [63] and Gray and Ancey [44], consider a mixture of particles of different sizes, but the same density, that flows down a chute inclined at an angle $\zeta$ to the horizontal. Let the mixture be divided into a discrete number of grain size classes, or constituents $\nu$, whose concentrations are denoted by $\phi^{\nu} \in[0,1]$ per unit solids volume fraction. The sum of the concentrations over all the solid phases is necessarily equal to unity, $\sum_{\forall \nu} \phi^{\nu}=1$, and the overall solids volume fraction is assumed to be constant and uniform throughout the mixture [55, 64-66]. A coordinate system $O x y z$ is defined with the $x$-axis pointing down the chute, the $y$-axis pointing across the chute and the $z$-axis being the upward pointing normal as shown in figure 1(b). For each constituent $\nu$, mixture theory [67, 68] defines overlapping partial densities $\rho^{\nu}$, partial velocities $\mathbf{u}^{\nu}$ and partial pressures $p^{\nu}$. These partial quantities are related to corresponding (starred) intrinsic variables defined on the pure phase rather than the mixture. In standard mixture theory, the partial and intrinsic velocity fields are identical, but the other fields such as the density, Cauchy stresses and pressures are usually related by a linear volume fraction scaling

$$
\rho^{\nu}=\phi^{\nu} \rho^{\nu *}, \quad \boldsymbol{\sigma}^{\nu}=\phi^{\nu} \boldsymbol{\sigma}^{\nu *}, \quad p^{\nu}=\phi^{\nu} p^{\nu *}, \quad \mathbf{u}^{\nu}=\mathbf{u}^{\nu *}
$$

The bulk density $\rho=\sum_{\forall \nu} \rho^{\nu}$ and the bulk pressure $p=\sum_{\forall \nu} p^{\nu}$ are defined by the sum of the partial densities and partial pressures. Each constituent satisfies individual mass and momentum balances

$$
\begin{gathered}
\frac{\partial \rho^{\nu}}{\partial t}+\nabla \cdot\left(\rho^{\nu} \mathbf{u}^{\nu}\right)=0 \\
\frac{\partial}{\partial t}\left(\rho^{\nu} \mathbf{u}^{\nu}\right)+\nabla \cdot\left(\rho^{\nu} \mathbf{u}^{\nu} \otimes \mathbf{u}^{\nu}\right)=\nabla \cdot \boldsymbol{\sigma}^{\nu}+\rho^{\nu} \mathbf{g}+\boldsymbol{\beta}^{\nu},
\end{gathered}
$$

where $\otimes$ is the dyadic product and $\mathbf{g}$ is the gravitational acceleration vector. The interaction force $\boldsymbol{\beta}^{\nu}$ is the force exerted on phase $\nu$ by all the other constituents. These interaction forces sum to zero over all constituents $\sum_{\forall \nu} \boldsymbol{\beta}^{\nu}=\mathbf{0}$. The Cauchy stress $\boldsymbol{\sigma}^{\nu}=-p^{\nu} \mathbf{1}+\boldsymbol{\tau}^{\nu}$ is broken down into a spherical pressure $-p^{\nu} \mathbf{1}$ and a deviatoric stress $\tau^{\nu}$, where $\mathbf{1}$ is the unit tensor. It is assumed that in the normal direction the pressure dominates, and both the deviatoric stresses and the normal acceleration terms can be neglected. This implies that the normal components of the momentum balances (7) sum to

$$
\frac{\partial p}{\partial z}=-\rho g \cos \zeta
$$

over all constituents, where $g$ is the constant of gravitational acceleration. Since the bulk density is constant and the avalanche free-surface is traction free (at $z=s$ ), equation (8) can be integrated through the avalanche depth, subject to the condition that $p(s)=0$, to show that the pressure is lithostatic $p=$ $\rho g(s-z) \cos \zeta$. Gray and Thornton [54] observed that small particles support less of the overburden pressure as they percolate downwards, and that the large grains must consequently support more of the load. This 
led them to introduce a factor $f^{\nu} \in[0,1]$, which determines the proportion of the lithostatic pressure carried by constituent $\nu$, i.e.

$$
p^{\nu}=f^{\nu} p
$$

Summing the partial pressure (9) over all constituents implies that $\sum_{\forall \nu} f^{\nu}=1$. The interaction drag law proposed by Gray and Chugunov[63] consists of three terms

$$
\boldsymbol{\beta}^{\nu}=p \nabla f^{\nu}-\rho^{\nu} c\left(\mathbf{u}^{\nu}-\mathbf{u}\right)-\rho d \nabla \phi^{\nu}
$$

65

where $c$ is the coefficient of inter-particle drag, $d$ is the coefficient of diffusive remixing and $\mathbf{u}$ is the barycentric or bulk velocity, defined by $\rho \mathbf{u}=\sum_{\forall \nu} \rho^{\nu} \mathbf{u}^{\nu}$. The first term in (10) combines with the pressure gradient in (7) to ensure that the percolation process is driven by the intrinsic rather than the partial pressure gradient, the second term provides a linear resistance to relative motion and the third term models diffusive remixing of the particles. The interaction $\operatorname{drag} \boldsymbol{\beta}^{\nu}$ has been constructed so that it automatically satisfies the summation 70 condition $\sum_{\forall \nu} \boldsymbol{\beta}^{\nu}=\mathbf{0}$.

\section{Segregation flux functions}

The bulk velocity $\mathbf{u}$ has components $u, v$ and $w$ in the downslope, cross-slope and normal directions, respectively. In the down and cross-slope directions the constituent velocities, $u^{\nu}$ and $v^{\nu}$, are assumed to be equal to the bulk velocity, i.e. $u^{\nu}=u$, and $v^{\nu}=v$, while the constituent velocity in the normal direction can be calculated by substituting (5), (8), (9) and (10) into the normal component of the momentum balance (7). Assuming that the normal accelerations are negligible, this implies that

$$
\phi^{\nu} w^{\nu}=\phi^{\nu} w+\left(f^{\nu}-\phi^{\nu}\right)(g / c) \cos \zeta-(d / c) \frac{\partial \phi^{\nu}}{\partial z}
$$

In the absence of diffusion, a particle will be squeezed upwards relative to the bulk if $f^{\nu}>\phi^{\nu}$, it will percolate downwards if $f^{\nu}<\phi^{\nu}$ and there will be no net motion if $f^{\nu}=\phi^{\nu}$. Gray and Thornton [54] argued that if there were no particles from a particular constituent then that phase could not carry any of the load and if the mixture was composed entirely of that phase then it must carry all of the load. This imposes two constraints on each constituent

$$
f^{\nu}=0, \quad \text { when } \quad \phi^{\nu}=0, \quad \text { and } \quad f^{\nu}=1 \text {, when } \phi^{\nu}=1 .
$$

For a bidisperse mixture composed of large $(\nu=l)$ and small $(\nu=s)$ particles the simplest functions that satisfy the constraints (12) have a quadratic dependence on the local concentration of each constituent, i.e.

$$
f^{l}=\phi^{l}+B_{l s} \phi^{l} \phi^{s}, \quad f^{s}=\phi^{s}-B_{l s} \phi^{l} \phi^{s}
$$

where $B_{l s}$ determines the perturbation of $f^{l}$ away from $\phi^{l}$ due to the presence of the small grains and hence the magnitude of the pressure perturbation away from lithostatic in (9). Note that the form (13) automatically satisfies the summation constraint $\sum_{\forall \nu} f^{\nu}=1$.

One of the beauties of the mixture theory approach is that it is possible to change the model assumptions to investigate different effects within the same general framework. Thornton et al. [55] derived an equivalent segregation flux to (11) using a three-phase model that accounted for an inviscid interstitial fluid with intrinsic density $\rho^{f *}$ that differed from the density of the grains $\rho^{g *}$. They showed that with this formulation there was an extra factor $\hat{\rho}=\left(\rho^{g *}-\rho^{f *}\right) / \rho^{g *}$ that pre-multiplied the term $\left(f^{\nu}-\phi^{\nu}\right)$ in (11). The additional o factor $\hat{\rho}$ is equivalent to a buoyancy force on the grains that reduces their propensity to segregate, e.g. if the density of the particles and the fluid are equal then segregation is suppressed completely [11, 53]. The flux functions have also recently been extended to include particle-size and particle density segregation [69, 70].

For a bidisperse mixture, Gajjar and Gray [71] proposed a more complicated higher order dependence of $f^{\nu}$ on the concentration

$$
f^{l}=\phi^{l}+B_{l s} F\left(\phi^{s}\right), \quad f^{s}=\phi^{s}-B_{l s} F\left(\phi^{s}\right)
$$


where $F$ is a general positive function satisfying

$$
F(0)=0, \quad F(1)=0 .
$$

Gajjar and Gray [71] considered $F\left(\phi^{s}\right)=A_{\gamma} \phi^{s}\left(1-\phi^{s}\right)\left(1-\gamma \phi^{s}\right)$, where $\gamma$ is a skewness parameter and the factor $A_{\gamma}$ is chosen so that the maximum is equal to the quadratic case. This latter asymmetric flux function was introduced to model the experimental observation that a single small particle percolates down through a matrix of large particles faster than a single large particle is squeezed up through a matrix of fines [72, 73]. For $\gamma \in(0.5,1]$ this flux function becomes non-convex and the maximum large-particle rise velocity occurs at $\phi_{c r i t}^{s}$, i.e. a group of large particles may rise faster than an isolated large grain. Bridgwater et al.'s [49] original segregation equation (1) also had a non-convex cubic flux $F\left(\phi^{s}\right)=\phi^{s}\left(1-\phi^{s}\right)^{2}$.

Fan and Hill [15, 74] modified the derivation for shear-induced segregation. Here the kinetic stress associated with velocity fluctuation correlations (granular temperature) drives all particles toward regions of low shear rate and combines with a kinetic sieving effect in which small particles are more likely than large particles to find voids into which they can travel. As a result small particles segregate to regions of low shear rate and squeeze large particles in the opposite direction. The resulting model has the same $\phi^{s}\left(1-\phi^{s}\right)$ structure in the segregation term, but is driven by gradients of the kinetic stresses associated with velocity fluctuations rather than by gravity. Large particles therefore move towards regions of high granular temperature. This model can therefore explain the motion of large particles to the walls of a vertical chute flow and their rise to the surface of an avalanche in a rotating drum [16]. However, it is not yet clear that it can fully explain the rise of large particles to the surface of an avalanche in chute flow [75], where high granular temperatures develop near the base of the flow [64] rather than at the surface. If the kinetic stresses do turn out to be the critical driving forces for segregation then it will be necessary to compute them as part of the solution for the bulk flow [76], which significantly complicates the model.

Gray and Ancey [44] showed that it is possible to generalize the bidisperse model to any number of discrete grain-size classes. For the quadratic flux functions this is done by taking an additive decomposition of the bidisperse perturbations, i.e.

$$
f^{\nu}=\phi^{\nu}+\sum_{\forall \mu} B_{\nu \mu} \phi^{\nu} \phi^{\mu}
$$

where the non-dimensional parameter $B_{\nu \mu}$ determines the magnitude of the pressure perturbation for constituent $\nu$ due to the presence of constituent $\mu$. This form automatically satisfies the constraints (12) and reduces to the bidisperse case in any two-component sub-mixture. Assuming that there are no pressure perturbations exerted by any constituent on itself, and that the pressure perturbation on constituent $\nu$ due to constituent $\mu$ is equal and opposite to the pressure perturbation on constituent $\mu$ by constituent $\nu$, then

$$
B_{(\nu \nu)}=0, \quad \forall \nu, \quad B_{\nu \mu}=-B_{\mu \nu}, \quad \forall \nu \neq \mu
$$

and the summation condition $\sum_{\forall \nu} f^{\nu}=1$ is automatically satisfied. The matrix $\mathbf{B}$ formed by the coefficients $B_{\nu \mu}$ has the interesting property that it is antisymmetric, i.e. $\mathbf{B}=-\mathbf{B}^{T}$, where the superscript $T$ denotes the transpose. More recently, Marks et al. [77] have generalized the flux functions and balance laws to continuous poly-disperse grain-size distributions, which is a significant extension of the theory.

\section{Derivation of a general segregation equation}

Following Gray and Ancey [44] it is convenient to introduce non-dimensional variables using the scalings

$$
x=L \tilde{x}, \quad z=H \hat{z}, \quad(u, v)=U(\hat{u}, \hat{v}), \quad\left(w, w^{\nu}\right)=(H U / L)\left(\hat{w}, \hat{w}^{\nu}\right), \quad t=(L / U) \hat{t},
$$

where $L$ is a typical downstream length scale, $H$ is a typical thickness and $U$ is a typical downstream velocity magnitude. An equation for the normal velocity of constituent $\nu$ can be obtained by substituting (16) into (11), dividing through by $\phi^{\nu}$ and applying the scalings to give

$$
w^{\nu}=w+\sum_{\forall \mu} S_{\nu \mu} \phi^{\mu}-D_{r} \frac{\partial}{\partial z}\left(\ln \phi^{\nu}\right),
$$


where the hats have been dropped for simplicity. The non-dimensional segregation rates $S_{\nu \mu}$ and the nondimensional diffusion coefficient $D_{r}$ are

$$
S_{\nu \mu}=\frac{L}{H U} q_{\nu \mu}, \quad D_{r}=\frac{L}{H^{2} U} D \quad \text { where } \quad q_{\nu \mu}=B_{\nu \mu}(g / c) \cos \zeta, \quad D=d / c .
$$

The matrix $\mathbf{S}$ formed by the segregation coefficients $S_{\nu \mu}$ is also antisymmetric (since it is proportional to $\mathbf{B}$ ) and $S_{\nu \mu}$ is positive if constituent $\nu$ is larger than constituent $\mu$. Substituting the normal velocity (19) into the mass balance equation (6) yields the non-dimensional segregation equation for phase $\nu$

$$
\frac{\partial \phi^{\nu}}{\partial t}+\nabla \cdot\left(\phi^{\nu} \mathbf{u}\right)=\frac{\partial}{\partial z}\left(\mathcal{F}^{\nu}\right)
$$

where the flux in the normal direction

$$
\mathcal{F}^{\nu}=-\sum_{\forall \mu} S_{\nu \mu} \phi^{\nu} \phi^{\mu}+D_{r} \frac{\partial \phi^{\nu}}{\partial z}
$$

accounts for the segregation and diffusion of the particles. For a given bulk velocity field $\mathbf{u}$, equation (21) must be solved subject to no flux conditions at the surface and the base of the avalanche

$$
\mathcal{F}^{\nu}=0, \quad \text { at } \quad z=s(x, y, t) \quad \text { and } \quad z=b(x, y, t),
$$

respectively. Equation (21) has been carefully constructed to ensure that when it is summed over all constituents it yields the bulk incompressibility condition $\nabla \cdot \mathbf{u}=0$. This condition and the lithostatic pressure distribution (8), are the key assumptions underlying most models for granular avalanches and geophysical mass flows [e.g 1, 20, 21, 30, 78-84]. The bulk three-dimensional velocity field $\mathbf{u}$ may be reconstructed from depth-averaged avalanche models [e.g. 58], or it may also be computed directly using the $\mu(I)$ rheology [85].

\section{Segregation in bidisperse mixtures}

For a bidisperse mixture of large and small particles there is a single positive segregation rate $S_{l s}$, since $S_{s l}=-S_{l s}$. The general theory of Gray and Ancey [44], given by equations (21)-(22), yields two segregation equations for the large and small particles

$$
\begin{aligned}
\frac{\partial \phi^{l}}{\partial t}+\nabla \cdot\left(\phi^{l} \mathbf{u}\right)+\frac{\partial}{\partial z}\left(S_{l s} \phi^{l} \phi^{s}\right) & =\frac{\partial}{\partial z}\left(D_{r} \frac{\partial \phi^{l}}{\partial z}\right), \\
\frac{\partial \phi^{s}}{\partial t}+\nabla \cdot\left(\phi^{s} \mathbf{u}\right)-\frac{\partial}{\partial z}\left(S_{l s} \phi^{s} \phi^{l}\right) & =\frac{\partial}{\partial z}\left(D_{r} \frac{\partial \phi^{s}}{\partial z}\right) .
\end{aligned}
$$

The summation condition $\sum_{\forall \nu} \phi^{\nu}=1$ implies $\phi^{l}=1-\phi^{s}$ and hence only a single segregation equation needs to be solved. It is conventional to solve for the small particles using (25), which becomes

$$
\frac{\partial \phi^{s}}{\partial t}+\nabla \cdot\left(\phi^{s} \mathbf{u}\right)-\frac{\partial}{\partial z}\left(S_{l s} \phi^{s}\left(1-\phi^{s}\right)\right)=\frac{\partial}{\partial z}\left(D_{r} \frac{\partial \phi^{s}}{\partial z}\right) .
$$

This is essentially the same as the form written down directly by Dolgunin and Ukolov [60] and given in equation (4). The only difference is that a full vector velocity field $\mathbf{u}$ is used to transport the grains rather than just the downstream component. On the basis that diffusive remixing of the grains is an isotropic process with no preferential direction [53], Fan et al. [86] have suggested generalizing the diffusive term to $\nabla \cdot\left(D_{r} \nabla \phi^{\nu}\right)$. However, because the $x$ and $y$ derivatives are generally small compared to gradients in the $z$ direction, the lateral gradients in the diffusion term can usually be neglected.

For problems that are uniform in the $x$ and $y$ directions, Gray and Chugunov [63] showed that it was possible to use the Cole-Hopf transformation to construct exact one-dimensional time-dependent solutions 
for arbitrary initial conditions. They compared the one-dimensional exact solution for the fully developed steady-state to Monte Carlo simulations of Khakhar et al. [2] and obtained good agreement with segregation Péclet numbers $P e$ (the ratio of the segregation rate $S_{l s}$ to the diffusion rate $D_{r}$ ) between 4 and 9 . Marks and Einav [87] developed a very simple cellular automaton model for the segregation process, based on large and small particles exchanging positions with a given probability and frequency, and subject to the constraints that large particle could only rise and fines could only fall. By averaging over many realizations they showed that that this was consistent with hyperbolic continuum theory of Gray and Thornton [54] in which the segregation rate $S_{l s}$ was dependent on the shear-rate. Thornton et al. [88] obtained very good agreement between the diffuse steady-state solution [63] and three-dimensional Discrete Particle Method (DPM) simulations with spheres. By fitting the solution to the simulated results they determined the functional dependence of the Péclet number on the grain size ratio. This shows that even small differences in the size of the particles leads to segregation with a rapid rise in Péclet number to seven at a grain-size ratio of 1.7. Above this value the Péclet number appears to saturate or even decrease slightly. More recently, Staron and Phillips [19] have studied the segregation time-scale using DPM simulations with disks and determined that it scales linearly with the small particle concentration. This is supportive of the asymmetric flux functions of Gajjar and Gray [71] and matches the experimental observations of van der Vaart et al. [73]. In addition, Fan and co-workers [86, 89, 90] have studied segregation in two-dimensional bounded heaps with fluid and solid-like regions. An incompressible bulk velocity field is determined from DPM simulations, and subsequently used in the computation of the resulting grain-size distribution using the bidisperse segregation equation (26) with an extended diffusive term. This approach produces very good results that explain the segregation pattern in heaps in a continuously depositing regime, but it is unable to capture flow transitions that lead to stratification patterns $[3,7,58,91]$.

While the Discrete Particle Method (DPM) provides a rich opportunity to study the details of the segregation process for spheres and disks, it should not be forgotten that these are models in themselves with a wide variety of contact laws and other assumptions. It is, therefore, vitally important to compare the theory with real experimental data. However, experiments in this area still remain extremely challenging, because it is very difficult to determine the evolving concentration in an opaque and rapidly moving flow. Savage and Lun's [10] movable hopper and splitter plate experiment provides one of the best data sets, but it is relatively low resolution with only five data points through the avalanche depth. Another approach is that of Wiederseiner et al. [92] who used a high speed camera to take pictures through a transparent sidewall. By averaging 2000 frames and stitching together the images at 10 downstream locations a complete picture (Fig. 2a) was built up along the chute. Despite the limitations of only observing the segregation at the sidewall, they were able to use a calibration curve to map the raw grey-scale data into a concentration distribution (Fig. 2b) with darker regions corresponding to higher concentrations of large particles. The flow was of almost constant depth $H=24 \mathrm{~mm}$ and the measured velocity profile was to a good approximation equal to $u(z)=\lambda \bar{u} \exp (\lambda z / h) /(\exp (\lambda)-1)$ where the depth-averaged velocity $\bar{u}=32.5 \mathrm{~mm} / \mathrm{s}$ and $\lambda=3.24$ as shown in figure $2(\mathrm{~d})$. At the inflow all the large particles were placed at the base of the flow with fines on top. Sufficiently far downstream this distribution reversed and was somewhat diffuse. Since the depth-averaged influx of small particles was known $(\bar{\phi}=0.74)$ the one-dimensional steady state solution could be used to approximate the solution at $L=70 \mathrm{~cm}$ from the inflow as shown in figure 2(e) with a Péclet number equal to 19. Wiederseiner et al. [92] used a standard Galerkin finite element method programmed in the pdepe routine in Matlab to solve the two-dimensional steady-state segregation equation (26) and hence determine an appropriate segregation rate $S_{l s}$ to match the experimental data. This solution is shown in figure 2(c) and captures the key features of the flow. In particular, large particles enter in the slowly moving basal region of the flow and occupy most of the depth, but at $70 \mathrm{~cm}$ they are concentrated into a very thin surface thinner layer when the large particles are in the faster moving near-surface layers of the flow.

For slope inclinations close to the limit for flow, Wiederseiner et al.'s [92] experiments show that the Péclet number can rise to between 10 and 20, so the limit of no diffusion is a useful approximation. When $D_{r}=0$ it is possible to use the method of characteristics to construct two-dimensional steady-state [54, 55, 71] as 
(a)

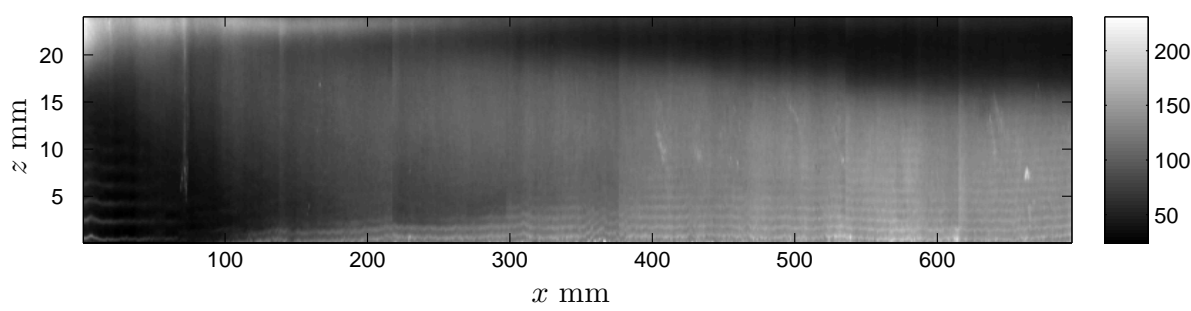

(b)
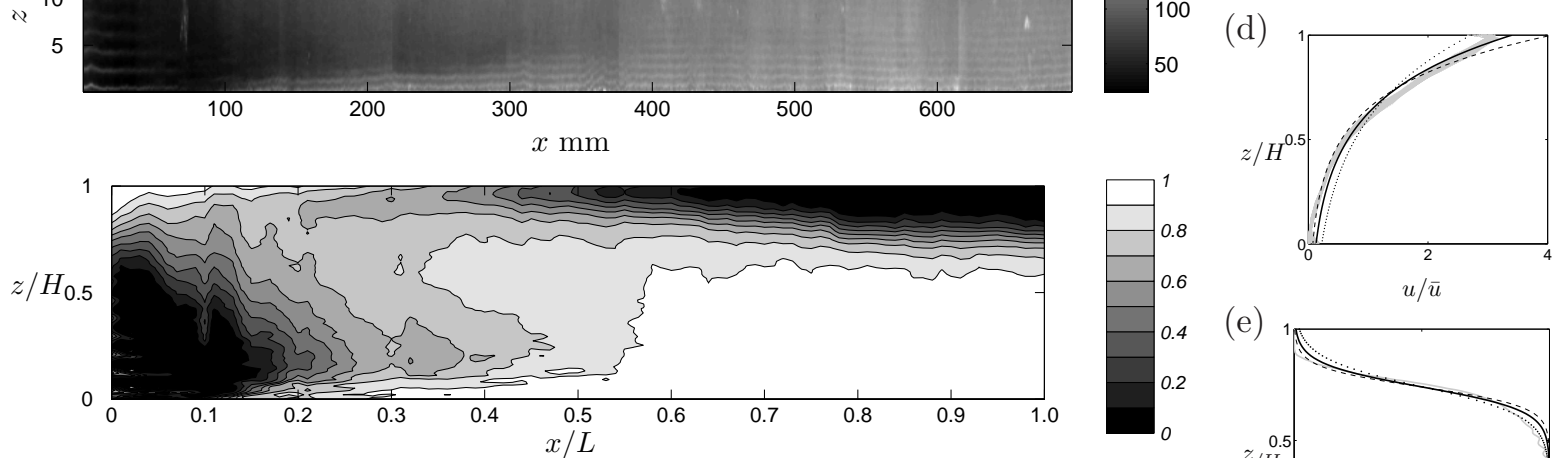

(c)
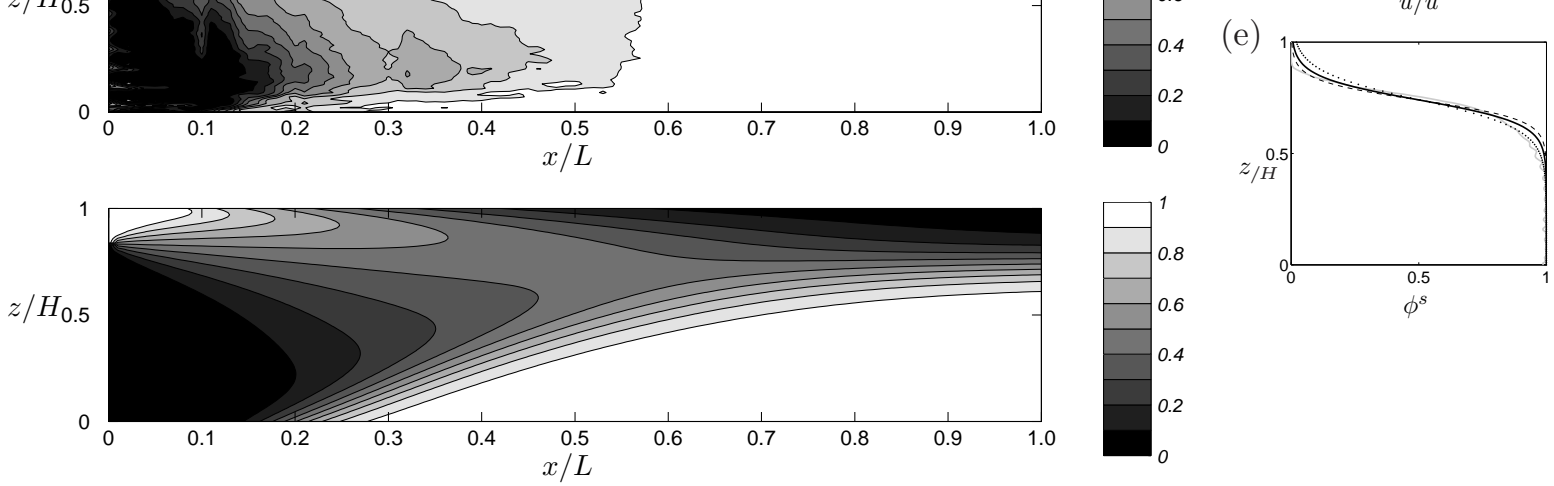

Figure 2: Raw image of the segregation process (a) obtained by averaging 2000 images at 10 downstream locations and stitching them together. Darker regions correspond to higher concentrations of large grains and lighter regions to higher concentration of fines (the sidebar on the right is the grey-level index, which ranges from 0 to 255 since the image was 8-bit encoded). The flow is from left to right with the inflow at $\mathrm{x}=0$. The height $H=24 \mathrm{~mm}$ the length $L=70 \mathrm{~cm}$ so the aspect ratio is 0.0343 , horizontal gradients are therefore much less than they might appear. A calibration curve is used to map the raw data to (b) a two-dimensional steady-state concentration field. Panel (c) shows the corresponding theoretical prediction with $q_{l s}=1.99 \mathrm{~mm} / \mathrm{s}$ and $D=2.52 \mathrm{~mm}^{2} / \mathrm{s}$. This uses a measured velocity field (d) which is approximated by an exponential profile $u(z)=\lambda \bar{u} \exp (\lambda z / h) /(\exp (\lambda)-1)$ with $\bar{u}=32.5 \mathrm{~mm} / \mathrm{s}$ and $\lambda=3.24$. The Péclet number is 19 which provides a very good fit (black solid line) to (e) the measured final steady state $70 \mathrm{~cm}$ downstream (grey solid line). (Reprinted with permission from Sebastien Wiederseiner, Nicolas Andreini, Gael Epely-Chauvin, Gaudenz Moser, Mathieu Monnereau, J.M.N.T. Gray, and Christophe Ancey (2011) Experimental investigation into segregating granular flows down chutes. Physics of Fluids 23, 013301. Copyright 2011, AIP Publishing LLC [92]).

Asymmetric flux functions [49, 69, 71, 73, 77] give rise to additional one-sided shocks (or semi-shocks) and adjacent expansion fans $[62,71]$, which significantly enhance the distance over which segregation occurs. A highlight of the theory is its ability to predict the formation of breaking size segregation waves, which form whenever small particles are sheared over large ones [22, 58, 93, 94]. These steadily travelling waves allow large particles near the base of the flow to be picked up and recirculated up into faster moving nearsurface layers [33]. This recirculation phenomenon is particularly important in the geophysical context, where it is fundamental to the formation of bouldery flow fronts [20, 23, 58, 95-98] and coarse-rich levees $[22,33-36,38,99-103]$.

Golick and Daniels [72] and May et al. [104] performed ring shear segregation experiments which showed that under a confining pressure both the velocity profile, $u=u(z)$, and the segregation rate, $q=q(z)$, could have a strong height dependence and exact solutions for this case have been constructed [104, 105]. Another interesting feature of these experiments is that they measured the degree of segregation based on the rise and fall of the top plate, which was caused by the grains packing denser in a mixed state than in a pure phase. While this is a relatively small effect in bidisperse mixtures composed of grains are not too issimilar in size, it can become significant in polydisperse systems with a wide grain-size distribution. Very high solids volume fractions can certainly be achieved in static systems [106], but how tightly particles can pack in sheared segregating systems is an open question. It is one that is, however, of interest to industry who wish to prevent very dense packings in consumer products such as washing powder. 


\section{Segregation in three-component mixtures}

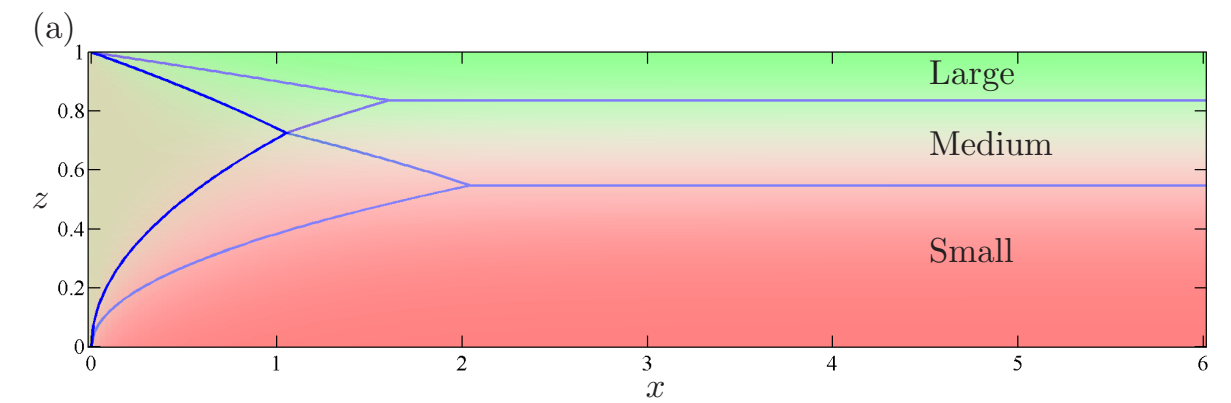
two coupled equations for the large and small particle concentrations

$$
\begin{aligned}
\frac{\partial \phi^{l}}{\partial t}+\nabla \cdot\left(\phi^{l} \mathbf{u}\right) & =\frac{\partial}{\partial z}\left(-S_{l m} \phi^{l}\left(1-\phi^{l}-\phi^{s}\right)-S_{l s} \phi^{l} \phi^{s}\right)+\frac{\partial}{\partial z}\left(D_{r} \frac{\partial \phi^{l}}{\partial z}\right), \\
\frac{\partial \phi^{s}}{\partial t}+\nabla \cdot\left(\phi^{s} \mathbf{u}\right) & =\frac{\partial}{\partial z}\left(S_{l s} \phi^{s} \phi^{l}+S_{m s} \phi^{s}\left(1-\phi^{l}-\phi^{s}\right)\right)+\frac{\partial}{\partial z}\left(D_{r} \frac{\partial \phi^{s}}{\partial z}\right)
\end{aligned}
$$
segregation of the ternary mixture in figure 1 although there is no mass exchange in this case.

Gray and Ancey [44] showed that for a three constituent mixture, composed of large, medium and small particles, denoted by the letters $\nu=l, m, s$, there are three independent positive segregation rates $S_{l m}, S_{l s}$ and $S_{m s}$. The antisymmetry property implies that the remaining segregation coefficients are $S_{m l}=-S_{l m}$, $S_{s l}=-S_{l s}$ and $S_{s m}=-S_{m s}$. The theory [44] yields three segregation equations, one of which can be eliminated by the summation condition $\sum_{\forall \nu} \phi^{\nu}=1$. Choosing to substitute for $\phi^{m}=1-\phi^{l}-\phi^{s}$ then yields

The steady-state spatially evolving problem with a prescribed velocity field $u(z)=2 z$ can be solved using a standard Galerkin finite element method e.g. pdepe in Matlab as shown in figure 3(a). A homogeneous mixture of concentrations $\phi_{0}^{l}=0.3, \phi_{0}^{s}=0.3$ and hence $\phi^{m}=0.4$ enters the chute at $x=0$ and sufficiently far downstream segregates into diffuse layers of large, medium and small particles coarsening upwards. This is known by geologists as reverse distribution grading. The solution shown in figure 3 is not unlike the

Figure 3: Steady-state segregation of a ternary mixture in a chute (a) from a homogenous inflow at $x=0$. The concentration of large, medium and small particles $\left(\phi^{l}, \phi^{m}\right.$, and $\left.\phi^{s}\right)$ can be read off the triangular contour scale (b). Blue lines show the position of concentration shocks in the absence of diffusion. This solution is for $S_{l s}=1, S_{l m}=4 / 8 S_{m s}=3 / 8$ and $D_{r}=0.05$ with initial concentrations $\phi_{0}^{l}=0.3, \phi_{0}^{s}=0.3$ and a velocity profile $u=2 z$. The blue lines are the position of concentration shocks in the absence of diffusion.

The blue lines in figure 3(a) represent an exact shock solution to the problem in the absence of diffusion. This provides a good approximation when $D_{r}$ is small and is useful in understanding the distance for complete segregation to occur. If $S_{l s}<S_{l m}$ and $S_{l s}<S_{m s}$ then for certain initial conditions the flow may become unstable. In the absence of diffusion the growth rate of the instability is unbounded, implying that the equations can be ill-posed [107]. Diffusion therefore plays a vital role in regularizing the theory.

\section{A depth-averaged segregation model}

Solving the bidisperse or poly-disperse size segregation equations in evolving three-dimensional flows still represents a considerable challenge. An alternative approach, first developed by Gray and Kokelaar [97, 98], is to integrate the segregation equation through the avalanche depth, so that it fits naturally into the depthaveraged framework of current avalanches models [e.g 1, 20, 21, 30, 78, 80, 81, 83]. For instance, neglecting cross-slope terms in (26) and using Leibniz' integral theorem, together with the surface and basal kinematic and no flux (23) conditions, implies that the depth-averaged segregation equation is

$$
\frac{\partial}{\partial t}(h \bar{\phi})+\frac{\partial}{\partial x}(h \overline{\phi u})=0,
$$



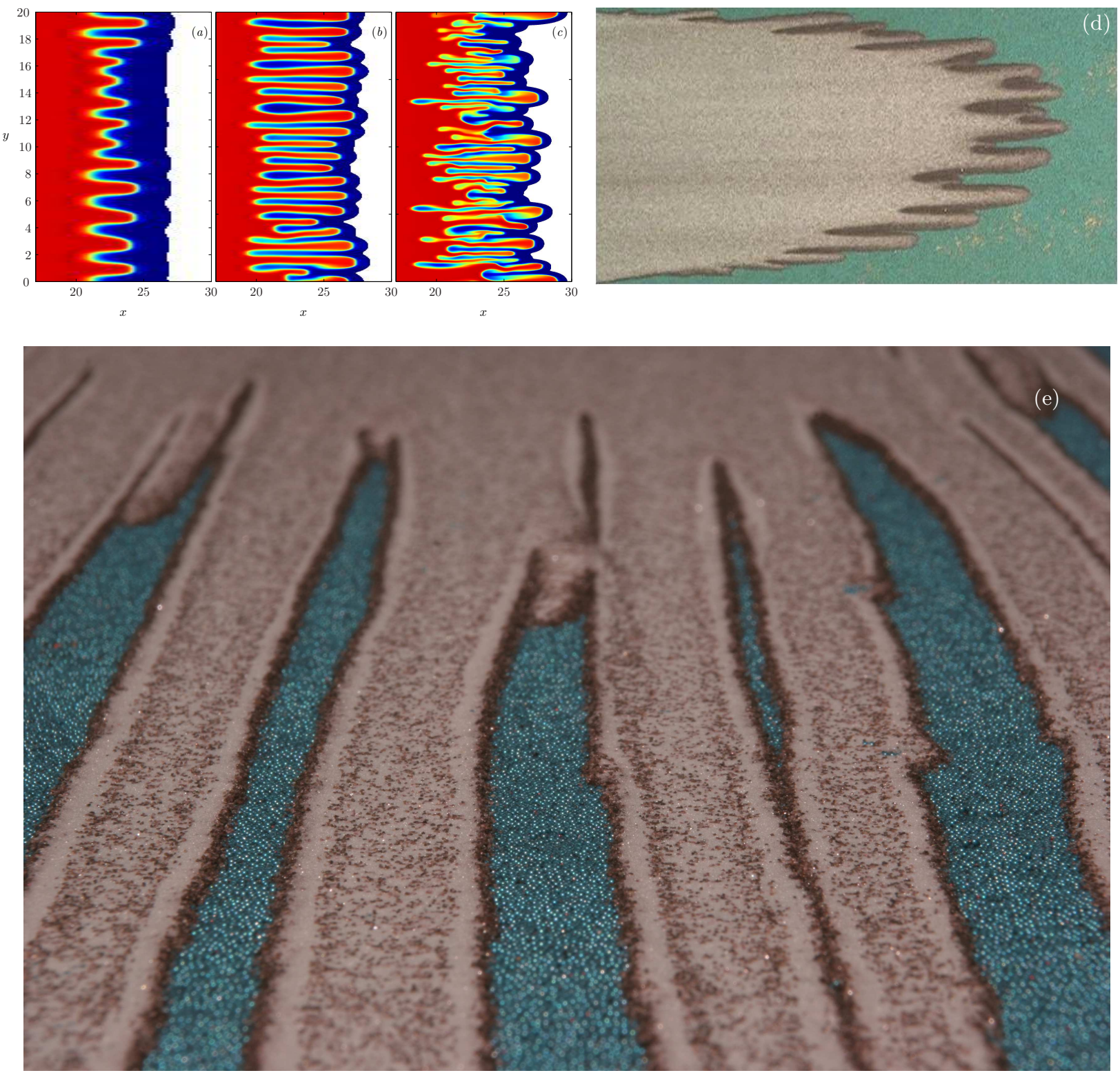

Figure 4: Contours of the depth-averaged concentration $\bar{\phi}$ as a function of downslope $x$ and cross-slope $y$ positions at time $t=90$ for three different sized computational grids (a) $500 \times 500$, (b) $1000 \times 1000$ and (c) $2000 \times 2000$. At the inflow the material enters with depth-averaged concentration $\bar{\phi}_{0}=0.9$ corresponding to red in the contour plots. Large particles are preferentially sheared towards the flow front and accumulate there before being shouldered aside to form static levees. These coarse rich regions are blue. An overhead photograph (d) of fingering of a bidisperse mixture of spherical (white) glass ballotini (75-150 $\mu \mathrm{m})$ and irregular (brown) carborundum grains $(315-350 \mu \mathrm{m})$ on a chute inclined at $27^{\circ}$ and made of monolayer of (turquoise) glass ballotini $(750-1000 \mu \mathrm{m})$. The image is approximately $57 \mathrm{~cm} \times 30 \mathrm{~cm}$ [M. J. Woodhouse, A. R. Thornton, C. G. Johnson, B. P. Kokelaar \& J. M. N. T. Gray (2012) Segregation-induced fingering instabilities in granular free surface flows, J. Fluid Mech. 709, 543-580 reprinted with permission 38]. Oblique head-on photograph of the final deposit (e) after the finite mass release has run-out and come to rest. Note the large particle rich (brown) levees are lined by $100 \%$ layer of fines (pure white). This relatively low-friction fines lining is progressively revealed as the inversely graded channelized flow drains down and comes to rest (speckled central band). Note that the image uses the depth of field to give an impression of distance, with regions that are far away or very close to the camera being out of focus. A movie of the experiment showing the laying down of the levees at the flow front is available in the supplementary material. 
where $h$ is the avalanche thickness, $\bar{\phi}$ is the depth-averaged concentration of small particles and $\overline{\phi u}$ is the depth integrated flux of small particles. Assuming that the avalanche is sharply inversely graded (all the large particles are on top of the fines) and that the downslope velocity is linear with depth, i.e.

$$
\phi^{s}=\left\{\begin{array}{ll}
0 & l \leq z \leq s, \\
1 & b \leq z \leq l,
\end{array} \quad \text { and } \quad u=\alpha \bar{u}+2(1-\alpha) \bar{u}\left(\frac{z-b}{h}\right), \quad 0 \leq \alpha \leq 1 .\right.
$$

where $\bar{u}$ is the depth-averaged downslope velocity, $z=b(x)$ is the height of the basal topography relative to the inclined plane [e.g. see 1] and $\alpha$ allows the velocity profile to vary from plug-flow $(\alpha=1)$ to simple shear $(\alpha=0)$, it follows that the depth-averaged quantities

$$
h \bar{\phi}=\int_{b}^{s} \phi^{s} d z=l-b=\eta, \quad h \overline{\phi u}=\int_{b}^{s} \phi^{s} u d z=\eta \bar{u}-(1-\alpha) \bar{u} \eta\left(1-\frac{\eta}{h}\right) .
$$

Substituting (31) into (29) and substituting $\eta=h \bar{\phi}$ implies that the depth-averaged segregation becomes

$$
\frac{\partial}{\partial t}(h \bar{\phi})+\frac{\partial}{\partial x}(h \bar{u} \bar{\phi})-\frac{\partial}{\partial x}((1-\alpha) h \bar{u} \bar{\phi}(1-\bar{\phi}))=0,
$$

which is remarkably similar to the segregation equation (26) from which it is derived. In particular, the form of the segregation term $\phi^{s}\left(1-\phi^{s}\right)$ in $(26)$ is very similar to the term $\bar{\phi}(1-\bar{\phi})$ in equation (32), hich immediately tells us that the depth-averaged equation also segregates particles, but laterally rather than vertically. Indeed, Gray and Kokelaar [97] called this the large particle transport equation, because it preferentially transports large particles towards the avalanche front, and allows them to recirculate there.

This equation opens up the possibility of coupling the basal friction of the bulk flow to the evolving particle size distribution, in order to model subtle segregation-mobility feedback effects. Figure $4(\mathrm{~d}, \mathrm{e})$ and the supplementary video show how these effects lead to the formation of coarse particle rich levees $[22,35]$ and flow fingers [33-36, 38, 39, 101-103]. As large irregular particles accumulate at the flow front, its frictional resistance to motion increases relative to the finer grained, more mobile material behind. This causes the front to become unstable and form a somewhat periodic series of extending fingers. The experiments in figure $4(\mathrm{~d}, \mathrm{e})$ show that each finger has an outer levee composed of almost $100 \%$ large (brown) carborundum particles and that these are lined internally by a layer of almost $100 \%$ small (white) spherical ballotini. This segregation-driven self-organization of the flow limits lateral spreading and minimizes the frictional resistance of the channel floor over which the fine particles of the (inversely graded) channel flow must shear. Thus by segregation-mobility feedback the flow is maintained in motion for much longer run-out distances than would otherwise be the case. Woodhouse et al. [38] coupled a two-dimensional generalization of equation (32) to a standard shallow-water type granular avalanche model [80] which consists of depth-averaged mass and momentum balance equations of the form

$$
\begin{aligned}
\frac{\partial h}{\partial t}+\nabla \cdot(h \overline{\mathbf{u}}) & =0, \\
\frac{\partial}{\partial t}(h \overline{\mathbf{u}})+\nabla \cdot(h \overline{\mathbf{u}} \otimes \overline{\mathbf{u}})+\nabla\left(\frac{1}{2} g h^{2} \cos \zeta\right) & =h g \mathcal{S},
\end{aligned}
$$

where $\nabla$ is the two-dimensional gradient operator, $\otimes$ is the two-dimensional dyadic product, '. ' is the twodimensional dot product and $\overline{\mathbf{u}}=(\bar{u}, \bar{v})$ is the two-dimensional depth-averaged velocity. The source term $\mathcal{S}=\sin \zeta \mathbf{i}-\mu(\overline{\mathbf{u}} /|\overline{\mathbf{u}}|) \cos \zeta$ is composed of the downslope component of gravitational acceleration and a resistive basal friction, where $\mathbf{i}$ is the unit vector in the downslope direction. The coupling of the depthaveraged avalanche equations and the depth-averaged segregation equation is achieved through the coefficient of basal friction $[34]$

$$
\mu=\mu^{L}(1-\bar{\phi})+\mu^{S} \bar{\phi}
$$

which is a depth-integrated concentration-weighted average of the large and small particle basal friction coefficients $\mu^{L}$ and $\mu^{S}$. Each set of particles is assumed to satisfy Pouliquen and Forterre's [108] extended 
friction law, which is rate dependent. When $\mu^{L}>\mu^{S}$ a resistive large-rich front develops that is shouldered to form a series of fingers as shown in figure 4(a). However, grid refinement studies [38] (shown in Fig. 4 a,b,c) indicate that the wavelength of the fingers is grid dependent, i.e. the numerical viscosity is setting their width. This is surprising since both the shallow water model and the segregation model are well-behaved in uncoupled simulations. Woodhouse et al. [38] showed that this was due to ill-posedness [107] when the characteristics of the two systems coincide and strict hyperbolicity is lost. Efforts are now underway to use the $\mu(I)$-rheology $[65,85]$ to introduce a depth-averaged viscous term [84] into the equations to set the wavelength.

\section{Outlook and challenges}

The early segregation theories of Bridgwater et al. [49], Savage and Lun [10] and Dolgunin and Ukolov [60] captured many of the essential ingredients of current bidisperse segregation models that have been derived using mixture theory $[15,54,55,63,71]$. Significant extensions of the theory include the ability to model particle-size and density driven segregation $[17,69,70,77,109]$ as well as polydisperse mixtures of grains $[44,77]$. These theories allow the particle-size distribution to be calculated as it evolves in both space and time, provided that the segregation and diffusion rates are known $\left(S_{l s}\right.$ and $D_{r}$ in the bidisperse case) and the bulk flow $\mathbf{u}$ is prescribed. Our physical understanding of the segregation process has been greatly helped by the construction of a wealth of exact solutions to the hyperbolic (non-diffuse) theory $[44,54,55,57,58,93]$. Time and spatially dependent diffuse solutions [63] can only be constructed by using the Cole-Hopf transformation for laterally uniform problems. However, the routine pdpde in Matlab provides a simple means of solving both the bidisperse and polydisperse segregation equations in simple configurations.

Discrete Particle Method (DPM) simulations [15, 16, 19, 88] provide a powerful means of testing the theory and of determining the functional dependence of the coefficients. While the agreement with the theory is good, the full functional dependence of the coefficients in the bi-disperse case is still open to debate and virtually nothing is known for the polydisperse case. DPM solutions should not be viewed as a substitute for real physical experiments, which are difficult and time consuming to perform. The simplest approach is to use a chute with a movable hopper and splitter plates to bin samples from various regions of the flow $[10,11,60]$. This gives good, but fairly low vertical resolution, with maybe only five or six data points through the avalanche depth. An alternative approach is that of Wiederseiner et al. [92] who filmed the evolving size distribution through the sidewall and used a calibration curve to infer the concentration. While this gives very high resolution data it necessarily suffers from sidewall effects [110]. It is anticipated that in future non-invasive techniques such as using indexed matched fluids [73, 111] or x-ray tomography [112] will be able to dramatically improve our understanding of the segregation process. Another major challenge for the future is to couple the size-segregation to the evolving flow field. While Woodhouse et al. [38] have made a first attempt at this by coupling the depth-averaged segregation equation [97] to a depth-averaged avalanche model [80] for the bulk flow, the field is completely open with regard to coupling non-depth-averaged theories to the bulk flow. This is difficult, not least because solving problems that involve fluid-solid granular phase transitions is a very tough problem even in mono-disperse systems $[85,113,114]$, without the added complexity of feedback from segregation. However, if segregation problems in rotating drums, heaps and silos $[7,58,86,89,90]$ are to be solved without prescribing the bulk flow this is an important problem that must be addressed.

\section{Acknowledgments}

This article was supported by NERC grants NE/E003206/1 and NE/K003011/1 as well as EPSRC grants $\mathrm{EP} / \mathrm{I} 019189 / 1$ and $\mathrm{EP} / \mathrm{K} 00428 \mathrm{X} / 1$.

\section{References}

[1] J. M. N. T. Gray, M. Wieland, K. Hutter, Proc. Roy. Soc. A 455 (1999) 1841-1874. 
[2] D. V. Khakhar, J. J. McCarthy, J. M. Ottino, Chaos 9 (1999) 594-610.

[3] S. C. Williams, Powder Technol. 2 (1968) 13-20.

[4] J. Baxter, U. Tüzün, D. Heyes, I. Hayati, P. Fredlund, Nature 391 (1998) 136.

[5] L. Bates, User guide to segregation, British Materials Handling Board, 1997.

[6] D. Schulze, Powders and Bulk Solids, Springer Berlin Heidelberg, 2008.

[7] J. M. N. T. Gray, K. Hutter, Continuum Mech. \& Thermodyn. 9 (1997) 341-345.

[8] K. M. Hill, D. V. Kharkar, J. F. Gilchrist, J. J. McCarthy, J. M. Ottino, Proc. Natl Acad. Sci. USA 96 (1999) 11701-11706.

[9] D. V. Khakhar, Macromolecular Materials and Engineering 296 (2011) 278-289.

[10] S. B. Savage, C. K. K. Lun, J. Fluid Mech. 189 (1988) 311-335.

[11] J. W. Vallance, S. B. Savage, in: A. D. Rosato, D. L. Blackmore (Eds.), IUTAM Symposium on segregation in granular materials, Kluwer, 2000, pp. 31-51.

[12] T. Shinbrot, F. J. Muzzio, Phys. Rev. Lett. 81 (1998) 4365-4368.

[13] G. V. Middleton, in: J. Lajoie (Ed.), Flysch Sedimentology in North America, Business and Economics Science Ltd, Toronto, 1970, pp. 253-272.

[14] G. V. Middleton, M. A. Hampton, in: D. J. Stanley, D. J. P. Swift (Eds.), Marine sediment transport and environmental management, Wiley, 1976, pp. 197-218.

[15] Y. I. Fan, K. M. Hill, New J. of Phys. 13 (2011) 095009.

[16] K. M. Hill, S. D. Tan, J. Fluid Mech. 756 (2014) 54-88.

[17] A. Tripathi, D. V. Khakhar, J. Fluid Mech. 717 (2013) 643-669.

[18] F. Guillard, Y. Forterre, O. Pouliquen, Phys. Fluids 26 (2014) 043301.

[19] L. Staron, J. C. Phillips, Phys. Fluids 26 (2014) 033302.

[20] R. M. Iverson, Rev. Geophys. 35 (1997) 245-296.

[21] R. M. Iverson, R. P. Denlinger, J. Geophys. Res. 106 (2001) 553-566.

[22] C. G. Johnson, B. P. Kokelaar, R. M. Iverson, M. Logan, R. G. LaHusen, J. M. N. T. Gray, J. Geophys. Res. 117 (2012) F01032.

[23] B. Turnbull, E. T. Bowman, J. N. McElwaine, Comptes Rendus Physique (2015).

[24] M. J. Branney, B. P. Kokelaar, Bull. Volcanol. 54 (1992) 504-520.

[25] D. M. Palladino, G. A. Valentine, J. Volcanol. Geotherm. Res. 69 (1995) 343-364.

[26] E. S. Calder, R. S. J. Sparks, M. C. Gardeweg, J. Volcan. Geotherm. Res. 104 (2000) $201-235$.

$310 \quad$ [27] H. U. Schminck, J. Sediment. Petrol. 37 (1967) 438-448.

[28] J. W. Vallance, in: H. Sigurdsson (Ed.), Encyclopedia of Volcanoes, Academic, 2000, pp. 601-616.

[29] S. G. Evans, O. Hungr, Canad. Geotech. J. 30 (1993) 620-636.

[30] S. B. Savage, K. Hutter, J. Fluid Mech. 199 (1989) 177-215.

[31] J. McElwaine, K. Nishimura, in: A. D. Rosato, D. L. Blackmore (Eds.), IUTAM Symposium on Segregation in Granular Flows, volume 81 of Solid Mechanics and Its Applications, Springer Netherlands, 2000, pp. 81-88.

[32] P. G. Rognon, F. Chevoir, H. Bellot, F. Ousset, M. Naam, P. Coussot, J. Rheol. 52 (2008) $729-748$.

[33] O. Pouliquen, J. Delour, S. B. Savage, Nature 386 (1997) 816-817.

[34] O. Pouliquen, J. W. Vallance, Chaos 9 (1999) 621-630.

[35] R. M. Iverson, J. W. Vallance, Geology 29 (2001) 115-118.

320 [36] G. Félix, N. Thomas, Earth \& Planetary Sci. Lett. 221 (2004) 197-213.

[37] F. Moro, T. Faug, H. Bellot, F. Ousset, Cold Regions Science and Technology 62 (2010) 55 - 66.

[38] M. J. Woodhouse, A. R. Thornton, C. G. Johnson, B. P. Kokelaar, J. M. N. T. Gray, J. Fluid Mech. 709 (2012) 543-580.

[39] B. P. Kokelaar, R. L. Graham, J. M. N. T. Gray, J. W. Vallance, Earth Planet. Sci. Lett. 385 (2014) $172-180$.

325 [40] L. T. Fan, Y. M. Chen, F. S. Lai, Powder Technol. 61 (1990) 255-287.

[41] J. J. McCarthy, Powder Technol. 192 (2009) 137-142.

[42] J. Bridgwater, Particuology 8 (2010) 563-567.

[43] B. Marks, A. Valaulta, A. Puzrin, I. Einav, in: A. Yu, K. Dong, R. Yang, S. Luding (Eds.), Powders and Grains 2013, volume 1542 of AIP Conf. Proc, pp. 658-661. 7th International Conference on Micromechanics of Granular Media (Powders and Grains), Sydney, AUSTRALIA, JUL 08-12, 2013.

[44] J. M. N. T. Gray, C. Ancey, J. Fluid Mech. 678 (2011) 535-588.

[45] D. V. Khakhar, A. V. Orpe, S. K. Hajra, Physica A 318 (2003) 129-136.

[46] J. R. Johanson, Chem. Eng. (1978) 183-188.

[47] J. M. N. T. Gray, AIP Conf. Proc. 1227 (2010) 343-362.

335 [48] B. A. Wills, Mineral Processing Technology, Pergamon, 1979. 
[49] J. Bridgwater, W. Foo, D. Stephens, Powder Technol. 41 (1985) 147.

[50] J. Bridgwater, Powder Technol. 15 (1976) 215236.

[51] A. M. Scott, J. Bridgwater, Industrial \& Engineering Chemistry Fundamentals 14 (1975) 22-27.

[52] J. Bridgwater, M. H. Cooke, A. M. Scott, T. I. Chem. Eng-Lond. 56 (1978) 157-167.

[53] J. Bridgwater, in: A. Mehta (Ed.), Granular Matter, Springer New York, 1994, pp. 161-193.

[54] J. M. N. T. Gray, A. R. Thornton, Proc. Roy. Soc. A 461 (2005) 1447-1473.

[55] A. R. Thornton, J. M. N. T. Gray, A. J. Hogg, J. Fluid Mech. 550 (2006) 1-25.

[56] J. M. N. T. Gray, M. Shearer, A. R. Thornton, Proc. Roy. Soc. A 462 (2006) 947-972.

[57] M. Shearer, J. M. N. T. Gray, A. R. Thornton, Eur. J. App. Math. 19 (2008) 61-86.

58] J. M. N. T. Gray, C. Ancey, J. Fluid Mech. 629 (2009) 387-423.

[59] P. Chadwick, Continuum Mechanics. Concise theory and problems, George Allen \& Unwin, 1976.

[60] V. N. Dolgunin, A. A. Ukolov, Powder Technol. 83 (1995) 95-103.

[61] G. J. Kynch, Trans. Faraday Soc. 48 (1952) 166-176.

[62] H. K. Rhee, R. Aris, N. R. Amundson, First-order partial differential equations: Volume 1 Theory and applications of single equations, Prentice-Hall, Englewood Cliffs, New Jersey, 1986.

[63] J. M. N. T. Gray, V. A. Chugunov, J. Fluid Mech. 569 (2006) 365-398.

[64] L. E. Silbert, D. Ertas, G. S. Grest, T. C. Halsey, D. Levine, S. J. Plimpton, Phys. Rev. E 64 (2001) 051302.

[65] GDR-MiDi, Eur. Phys. J. E 14 (2004) 341-365.

[66] P. G. Rognon, J. N. Roux, M. Naaim, F. Chevoir, Phys. Fluids 19 (2007) 058101.

355 [67] C. Truesdell, Rational Thermodynamics, Springer, 1984.

[68] L. W. Morland, Surveys in Geophysics 13 (1992) 209-268.

[69] D. R. Tunuguntla, O. Bokhove, A. R. Thornton, J. Fluid Mech. 749 (2014) 99-112.

[70] J. M. N. T. Gray, C. Ancey, J. Fluid Mech. (2015) Submitted.

[71] P. Gajjar, J. M. N. T. Gray, J. Fluid Mech. 757 (2014) 297-329.

72] L. A. Golick, K. E. Daniels, Phys. Rev. E 80 (2009) 042301.

[73] K. van der Vaart, P. Gajjar, G. Epely-Chauvin, N. Andreini, J. M. N. T. Gray, C. Ancey (2015) In prep.

[74] Y. Fan, K. M. Hill, Phys. Rev. Lett. 106 (2011) 218301.

[75] T. Weinhart, S. Luding, A. R. Thornton, AIP Conf. Proc. 1542 (2013) 1202-1205.

[76] M. Larcher, J. T. Jenkins, Phys. Fluids 25 (2013) 113301.

[77] B. Marks, P. Rognon, I. Einav, J. Fluid Mech. 690 (2012) 499-511.

[78] S. S. Grigorian, M. E. Eglit, I. L. Iakimov, Snow, Avalanches \& Glaciers. Tr. Vysokogornogo Geofizich Inst 12 (1967) 104-113.

[79] E. B. Pitman, C. C. Nichita, A. Patra, A. Bauer, M. Sheridan, M. Bursik, Phys. Fluids 15 (2003) $3638-3646$.

[80] J. M. N. T. Gray, Y. C. Tai, S. Noelle, J. Fluid Mech. 491 (2003) 161-181.

370 [81] A. Mangeney, F. Bouchut, N. Thomas, J. P. Vilotte, M. O. Bristeau, J. Geophys. Res. 112 (2007) F02017.

[82] O. Bokhove, A. R. Thornton, in: H. J. Fernando (Ed.), Handbook of Environmental Fluid Dynamics, Volume One: Overview and Fundamentals, CRC Press, 2012, pp. 545-556.

[83] X. Cui, J. M. N. T. Gray, J. Fluid Mech. 720 (2013) 314-337.

[84] J. M. N. T. Gray, A. N. Edwards, J. Fluid Mech. 755 (2014) 503-534.

375 [85] P. Jop, Y. Forterre, O. Pouliquen, Nature 44 (2006) 727-730.

[86] Y. Fan, C. P. Schlick, P. B. Umbanhowar, J. M. Ottino, R. M. Lueptow, J. Fluid Mech. 741 (2014) $252-279$.

[87] B. Marks, I. Einav, Granul. Matter 13 (2011) 211-214.

[88] A. Thornton, T. Weinhart, S. Luding, O. Bokhove, Int. J. Mod. Phys. C 23 (2012) 1240014.

[89] Y. Fan, Y. Boukerkour, T. Blanc, P. B. Umbanhowar, J. M. Ottino, R. M. Lueptow, Phys. Rev. E 86 (2012) 051305.

[90] Y. Fan, P. B. Umbanhowar, J. M. Ottino, R. M. Lueptow, Proc. Roy. Soc. A 469 (2013) 20130235.

[91] H. A. Makse, S. Havlin, P. R. King, H. E. Stanley, Nature 386 (1997) 379-382.

[92] S. Wiederseiner, N. Andreini, G. Epely-Chauvin, G. Moser, M. Monnereau, J. M. N. T. Gray, C. Ancey, Phys. Fluids 23 (2011) 013301.

385 [93] A. R. Thornton, J. M. N. T. Gray, J. Fluid Mech. 596 (2008) 261-284.

[94] M. Shearer, N. Giffen, Discrete \& Continuous Dyn. Sys. 27 (2010) 693-714.

[95] T. Takahashi, Journal of the Hydraulics Division, ASCE 106 (1980) 381-396.

[96] J. E. Costa, G. Williams, Debris flow dynamics, Technical Report 84-606, (videotape) U.S. Geological Survey, 1984.

[97] J. M. N. T. Gray, B. P. Kokelaar, J. Fluid Mech. 652 (2010) 105-137.

[98] J. M. N. T. Gray, B. P. Kokelaar, J. Fluid Mech. 657 (2010) 539. 
[99] T. C. Pierson, in: A. D. Abrahams (Ed.), Hillslope Processes, Allen and Unwin, Winchester, Mass., 1986, pp. 269-296.

[100] T. R. H. Davies, J. Hydrol. (NZ) 29 (1990) 18-46.

101] C. Goujon, B. Dalloz-Dubrujeaud, N. Thomas, Eur. Phys. J. E 23 (2007) 199-215.

[102] R. M. Iverson, M. Logan, R. G. LaHusen, M. Berti, J. Geophys. Res. 115 (2010) F03005.

[103] B. Cagnoli, G. P. Romano, J. Volcan. Geotherm. Res. 193 (2010) 18-24.

[104] L. B. H. May, L. A. Golick, K. C. Phillips, M. Shearer, K. E. Daniels, Phys. Rev. E 81 (2010) 051301.

[105] L. B. H. May, M. Shearer, K. E. Daniels, J. Nonlinear Sci. 20 (2010) 689-707.

[106] S. D. S. Reis, N. A. M. Araújo, J. S. Andrade, H. J. Herrmann, EPL 97 (2012) 18004.

[107] D. D. Joseph, J. C. Saut, Theoretical and Computational Fluid Dynamics 1 (1990) 191-227.

[108] O. Pouliquen, Y. Forterre, J. Fluid Mech. 453 (2002) 133-151.

[109] A. Tripathi, D. V. Khakhar, Phys. Rev. Lett. 107 (2011) 108001.

[110] P. Jop, Y. Forterre, O. Pouliquen, J. Fluid Mech. 541 (2005) 167-192.

[111] M. Harrington, J. H. Weijs, W. Losert, Phys. Rev. Lett. 111 (2013) 078001.

[112] S. McDonald, D. Harris, P. Withers, Int. J. Material Res. 103 (2012) 162-169.

[113] L. Staron, P.-Y. Lagrée, S. Popinet, Phys. Fluids 24 (2012) 103301.

[114] P.-Y. Lagrée, L. Staron, S. Popinet, J. Fluid Mech. 686 (2011) 378-408. 